(2) Open Access Full Text Article

\title{
Glycyrrhizic acid ameliorates myocardial ischemic injury by the regulation of inflammation and oxidative state
}

This article was published in the following Dove Press journal:

Drug Design, Development and Therapy

\section{Chongli $\mathrm{Xu}{ }^{1,2}$ \\ Caihong Liang ${ }^{2}$ \\ Weixin Sun ${ }^{3}$ \\ Jiandong Chen ${ }^{3}$ \\ Xiaohu Chen ${ }^{3}$}

'Nanjing University of Chinese Medicine, Nanjing 210029, People's Republic of China; ${ }^{2}$ Jiangnin Hospital of Nanjing, Nanjing 21 I I00, People's Republic of China; ${ }^{3}$ Affiliated Hospital of Nanjing University of Chinese Medicine, Nanjing University of Chinese Medicine, Nanjing 210023 , People's Republic of China
Correspondence: Xiaohu Chen Nanjing University of Chinese Medicine, I 38 Xianlin Avenue, Nanjing 210023 ,

People's Republic of China

Tel/fax+8625858। I I60

Email xhchennj@tom.com
Background: Glycyrrhizic acid (GA), a bioactive triterpenoid saponin isolated from the roots of licorice plants (Glycyrrhiza glabra), has been shown to exert a variety of pharmacological activities and is considered to have potential therapeutic applications. The purpose of the present study was to investigate the cardioprotective effect of GA on myocardial ischemia (MI) injury rats induced by isoproterenol (ISO), and explore the potential mechanisms underlying these effects.

Materials and methods: The rats were randomized into five groups: control, ISO, ISO+diltiazem (10 mg/kg), ISO+GA (10 mg/kg), and ISO+GA (20 mg/kg). Electrocardiogram and histopathological examination were performed. Markers of cardiac marker enzymes (creatine kinase-MB, lactate dehydrogenase), oxidative stress (superoxide dismutase, malondialdehyde [MDA]), and inflammation (TNF- $\alpha$, IL-1 $\beta$, and IL-6) were also measured in each group. Proteins involved in NF- $\mathrm{\kappa B}$ and Nrf-2/HO-1 pathway were detected by Western blot.

Results: GA decreased the ST elevation induced by MI, decreased serum levels of creatine kinase, lactate dehydrogenase, malondialdehyde, IL-6, IL-1 $\beta$, and TNF- $\alpha$, and increased serum superoxide dismutase and malondialdehyde activities. Furthermore, GA increased the protein

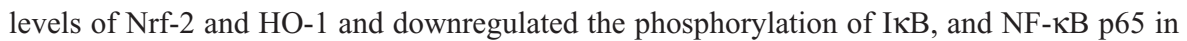
ISO-induced MI.

Conclusion: These observations indicated that GA has cardioprotective effects against MI, and these effects might be related to the activation of Nrf-2/HO-1 and inhibition of NF- $\mathrm{KB}$ signaling pathway in the myocardium.

Keywords: glycyrrhizic acid, myocardial ischemia, Nrf-2/HO-1, oxidative stress, inflammation

\section{Introduction}

In the past decade, cardiovascular diseases (CVDs) have been the major health issues worldwide, particularly in advanced countries. ${ }^{1}$ Ischemic heart diseases (IHDs) are the main form of CVDs, and could lead to 7.2 million deaths each year. ${ }^{2}$ At present, percutaneous coronary intervention (PCI) is effective in reducing the consequences of myocardial ischemia (MI) in patients with IHDs. However, PCI therapies also frequently cause reperfusion injury. Moreover, sudden cardiac death can occur before patients have the chance of receiving preventive PCI operation. Therefore, drug treatment is still a convenient and important strategy to rescue patients from acute myocardial infarction. ${ }^{3,4}$ Development of pharmacological agents effective against IHDs is mandatory. For IHDs, prolonged ischemia and subsequent reperfusion causes severe, irreversible damage to the myocardium, resulting in thrombolysis, angioplasty, coronary bypass, and heart transplantation..$^{5} \mathrm{MI}$ is multifactorial and includes the 
dysfunction of energy metabolism, robust oxygen radicals, calcium overload, and inflammatory responses. ${ }^{6}$ These factors lead to changes in myocardial functional status. Among the proposed factors, oxidative stress, which had been proved to be caused by reactive oxygen species (ROS) produced in ischemia conditions, ${ }^{7,8}$ is one of the most important mechanisms. Increased reactive oxygen and nitrogen species aggravate cell apoptosis, calcium overload, and inflammatory cytokine secretion, which could promote the progression of ischemia injury. Antioxidant molecules for reducing oxidative stress are deemed to be an ideal method to protect against MI injury. ${ }^{9}$ Nrf-2, expressed in cardiomyocytes, is an important endogenous antioxidative stress regulator. As a transcription factor, Nrf-2 plays a key role in preventing the development of myocardial dysfunction and the progression of cardiac remodeling. After being phosphorylated, Nrf-2 is translocated from the cytoplasm into cell nucleus to activate the Nrf-2-dependent antioxidant response, which includes HO-1. HO-1 possesses anti-inflammatory, antiapoptotic, antiproliferative, and especially cytoprotective effects. ${ }^{3}$ Its activation has been demonstrated to be related with many CVDs. ${ }^{10}$ It is thought to be an important potential target for curing some CVDs. Hence, upregulation of the Nrf-2/ HO-1 pathway may play a therapeutic role in attenuating MI injury.

Glycyrrhizic acid (GA), also known as glycyrrhizin, is one of the important active compounds isolated from the root of licorice. GA has a variety of pharmacological activities, such as being an anti-inflammatory, antiulcer, antiallergy, antioxidation, immune regulatory, antiviral, and anticancer agent, helping in liver protection, and so on. ${ }^{11}$ Furthermore, its promotion of Nrf-2 activity was also described in previous papers. ${ }^{12} \mathrm{GA}$ is reported to relieve cardiac ischemia in rats. ${ }^{13}$ However, it was not clear that whether GA attenuated MI injury through activating Nrf-2. Therefore, the purpose of present study was to investigate whether GA ameliorates MI injury, and to assess the role of Nrf2/ARE signaling pathway in the cardioprotective effect of GA.

\section{Materials and methods}

\section{Reagents}

GA was purchased from National Institutes for Food and Drug Control (Beijing, People's Republic of China). Diltiazem (Dit) was obtained from Simcere Pharmaceutical Group (Nanjing, People's Republic of China). Isoproterenol (ISO) hydrochloride injection was provided by Hongta Tobacco Group Company Limited (Yuxi, People's Republic of China). TNF- $\alpha$, IL-6, and IL-1 $\beta$ enzyme-linked immunosorbent assay
(ELISA) kits were supplied by Nanjing KeyGEN Biotech. Co., Ltd. (Nanjing, People's Republic of China). Malondialdehyde (MDA) and superoxide dismutase (SOD) commercial kits were purchased from Jiancheng Bioengineering Institute (Nanjing, People's Republic of China). Antibodies against $\mathrm{I} \kappa \mathrm{B}, \mathrm{p}-\mathrm{I} \kappa \mathrm{B}, \mathrm{NF}-\kappa \mathrm{B}$, and $\mathrm{p}-\mathrm{NF}-\kappa \mathrm{B}$ were produced by Cell Signaling Technology (Danvers, MA, USA).

\section{Animals}

Fifty adult male Sprague Dawley rats (weighing 200-250 g) were provided by the Comparative Medicine Centre of Yangzhou University. All the rats were maintained at a constant temperature of $25^{\circ} \mathrm{C} \pm 2^{\circ} \mathrm{C}$ in the Animal Center of China Pharmaceutical University. All the animals were kept on a $12 \mathrm{~h}$ light/ $12 \mathrm{~h}$ dark cycle and provided with standard water and food pellets ad libitum. All the experimental procedures were carried out in accordance with the National Institutes of Health Guidelines. All animal experiments were approved by the animal and ethics review committee of Nanjing University of Chinese Medicine, People's Republic of China.

\section{Experimental protocol}

Experimental rats were randomly divided into five groups ( $\mathrm{n}=10$ ) as follows: 1) control group, 2) ISO group, 3) ISO+Dit $(10 \mathrm{mg} / \mathrm{kg}$ ) group (positive control), 4) ISO+GA (10 mg/kg) group, and 5) ISO+GA (20 mg/kg) group. Rats in the administration groups were intraperitoneally pretreated with GA (10 and $20 \mathrm{mg} / \mathrm{kg}$ ) and Dit (10 mg/kg) for 7 days. Afterward, the animals in all groups, except for the control group, were administrated with ISO $(80 \mathrm{mg} / \mathrm{kg})$ by subcutaneous injection on two consecutive days. The rats in the control group were given equal volumes of saline at the same time. Twenty-four hours after ISO administration, the rats were anesthetized by $\mathrm{CO}_{2}$ asphyxiation, and electrocardiogram (ECG) was taken. After that, the animals were sacrificed, and the blood was collected and processed for further biochemical assays. The hearts tissue were excised and rinsed in ice-cold isotonic saline.

\section{ECG assessment}

The ECG were recorded with an ECG recording and analysis system (BL-420F, Chengdu TME Technology Company, Chengdu, People's Republic of China).

\section{Cardiac marker enzymes}

After the ECG detection, arterial blood samples were collected and centrifuged at 3,500 rpm for $15 \mathrm{~min}$ to obtain serum, and then stored at $-80^{\circ} \mathrm{C}$. Serum samples were thawed 
at room temperature before analysis. Myocardial damage was evaluated by measuring the serum levels of creatine kinase (CK-MB) and lactate dehydrogenase (LDH) with RT-9600 Semiautomatic Biochemical Analyzer (ShenZhenLeiDu Life Science, LLC, ShenZhen, People's Republic of China).

\section{Antioxidant enzymes assay}

The levels of oxidative stress markers, MDA, and the activities of antioxidants markers, SOD, were measured using commercial kits according to the manufacturer's protocols.

\section{Inflammation assay}

To explore the relationship between the cardioprotective effects of GA and the levels of inflammatory cytokines, IL-6, IL-1 $\beta$, and TNF- $\alpha$ levels in the serum were evaluated with ELISA kits. All experimental procedures were performed according to the kit manufacturer's instructions.

\section{Histological studies}

The fresh heart tissues (left cardiac apex, the ischemic part) were fixed in $10 \%$ formalin solution and embedded in paraffin for histopathological analysis. Then, the sections were processed for sectioning and stained with hematoxylin and eosin by standard histological methods. Afterward, the histopathological changes were examined by light microscopy (Nikon, Tokyo, Japan) at 200× magnification.

\section{Western blot analysis}

Heart tissues (left cardiac apex, the ischemic part) were homogenized in lysis buffer containing $1 \mathrm{~mL}$ of RIPA and $10 \mu \mathrm{L}$ of PMSF. The protein was quantified using BCA kit. Protein samples were loaded on 12\% SDS-PAGE and transferred to polyvinylidene fluoride membranes (Bio-Rad Laboratories, Hercules, CA, USA). The membranes were blocked with 5\% milk for $2 \mathrm{~h}$ and washed with TBST three times. The membranes were incubated with primary antibodies, which included antibodies against Nrf2 $(1: 1,000)$, HO-1 $(1: 1,000), \mathrm{p}-\mathrm{I} \kappa \mathrm{B}(1: 1,000), \mathrm{I} \kappa \mathrm{B}(1: 1,000), \mathrm{p}-\mathrm{NF} \kappa \mathrm{B}(1: 1,000)$, and NF- $\kappa \mathrm{B}(1: 1,000)$, overnight at $4^{\circ} \mathrm{C}$. After washing, the blots were incubated with HRP-labeled secondary antibodies for $2 \mathrm{~h}$. Immunoblots were visualized using the ECL detection system and a gel imaging system.

\section{Statistical analysis}

All statistical results are expressed as mean \pm SD. Significant differences were checked by one-way analysis of variance followed by Tukey multiple comparison tests. $p<0.05$ was considered significant.

\section{Results Effects of GA on ST-segment}

When the blood flow through the heart muscle is decreased by a partial or complete blockage of heart arteries, MI occurs and causes ST level changes in an ECG signal. A normal STsegment is characterized by its horizontal nature, whereas an abnormal ST-segment deviates from the horizontal path. ${ }^{13}$ In this study, $24 \mathrm{~h}$ after ISO administration, ST-segment elevation was recorded by lead II ECG. The ECG patterns in each group are shown in Figure 1. As seen in Figure 1, there was remarkable elevation in ST-segment in the model group compared with the control group, suggesting that the MI damage model has been established. The administration of GA induced a statistically significant reduction in STsegment elevation as compared with the vehicle group in a dose-dependent manner $(F(4,30)=21.43, p<0.01$ ) (Figures 1 and 2). These results reflect the positive effect of GA on cardiac function.

\section{Effects of GA on CK and LDH}

$\mathrm{CK}$ and $\mathrm{LDH}$ have been identified as essential diagnostic markers of myocardial injury and leak out from damaged myocardium to blood. ${ }^{14}$ Current studies measured the amounts of $\mathrm{CK}$ and $\mathrm{LDH}$ in serum to evaluate the myocardial damage. As shown in Figure 3, a marked increase in the levels of $\mathrm{CK}$ and LDH were detected in MI model group rats compared with those in the control group $(F(4,30)=76.56$, $p<0.01)$. But, compared with the model group, the GA significantly decreased serum $\mathrm{CK}$ and $\mathrm{LDH}$ at a dose of 10 and $20 \mathrm{mg} / \mathrm{kg}(F(4,30)=26.48, p<0.01)$.

\section{Effects of GA on SOD and MDA}

In this study, we evaluated the levels of SOD and MDA in the serum to explore the relationship between the cardioprotective effect of GA in MI and its antioxidant status. As shown in Figure 3, compared with control group, the model rats exhibited a significant increase in MDA content, while the level of SOD was significantly decreased, and those changes were reversed in GA and Dit groups $(\mathrm{SOD}, F(4,30)=12.33$, $p<0.01$; MDA, $F(4,30)=12.25, p<0.01)$. These results suggested the antioxidative stress effect of GA in alleviating MI injury.

\section{Effects of GA on TNF- $\alpha$, IL-I $\beta$, and IL-6}

Myocardial ischemic injury was characterized by increased levels of inflammatory factors such as TNF- $\alpha$, IL-1 $\beta$, and IL-6. To further evaluate and validate the protective function of GA during MI injury, we measured the levels of 


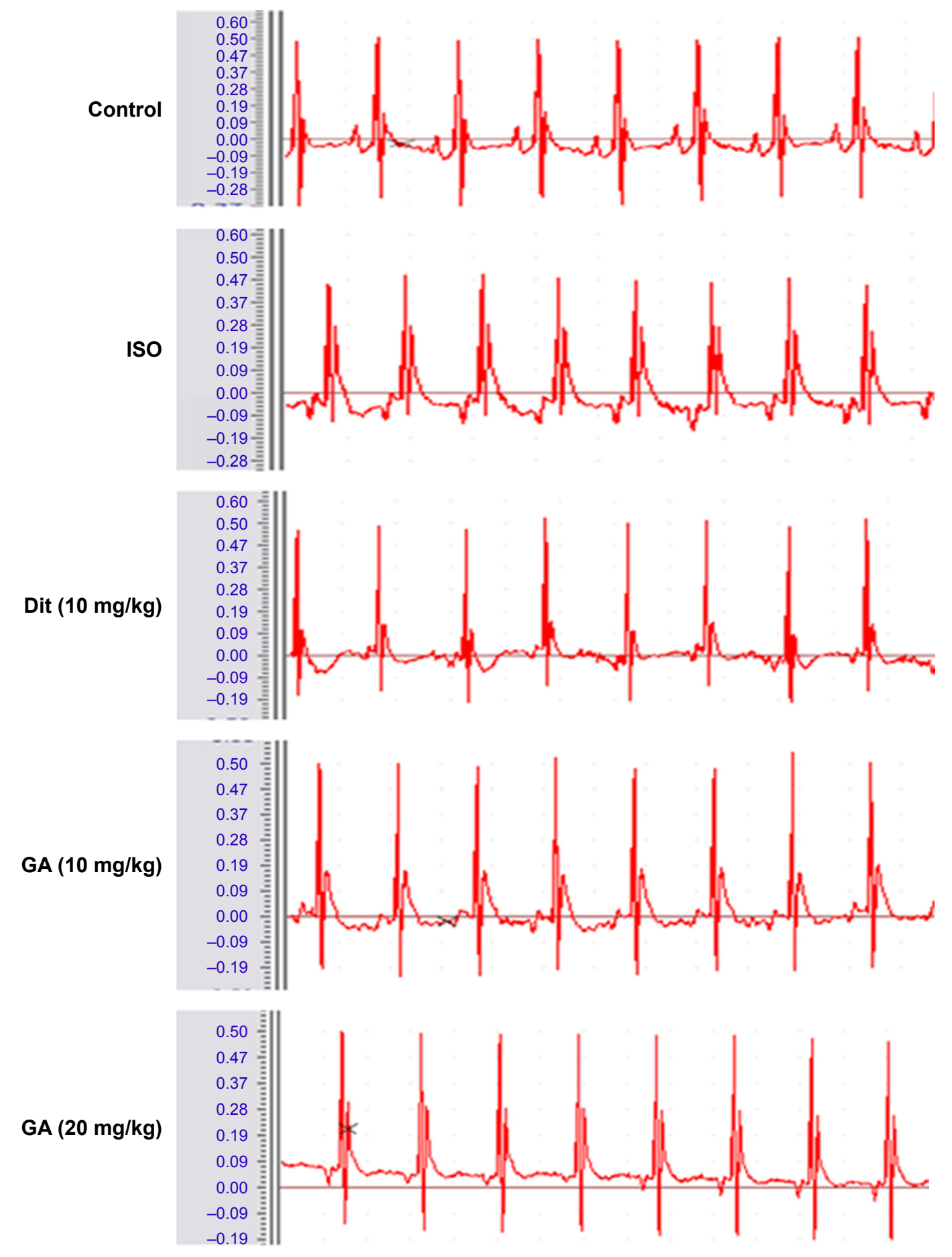

Figure I Effect of GA on ST-segment elevation.

Abbreviations: Dit, diltiazem; GA, glycyrrhizic acid; ISO, isoproterenol.

TNF- $\alpha$, IL- $1 \beta$, and IL- 6 in serum. It was noted that there was a marked increase in the levels of the TNF- $\alpha, \mathrm{IL}-1 \beta$, and IL- 6 as compared with that of rats in the control group $(\mathrm{TNF}-\alpha, F(4,30)=11.13, p<0.01$; IL-1 $\beta, F(4,30)=13.92$, $p<0.01$; IL-6, $F(4,30)=15.45, p<0.01$, Figure 4). Notably, the administration of GA (10 and $20 \mathrm{mg} / \mathrm{kg}$ ) and Dit could inhibit the increase, which indicated that GA and Dit significantly suppressed MI-induced inflammatory cytokine secretion in the serum.

\section{Effects of GA on myocardial histology}

Physiological impairment of myocardium tissue in the rats is illustrated in Figure 5. The myocardial tissues from control rat myocardium exhibited a regular integrity of myocardial 


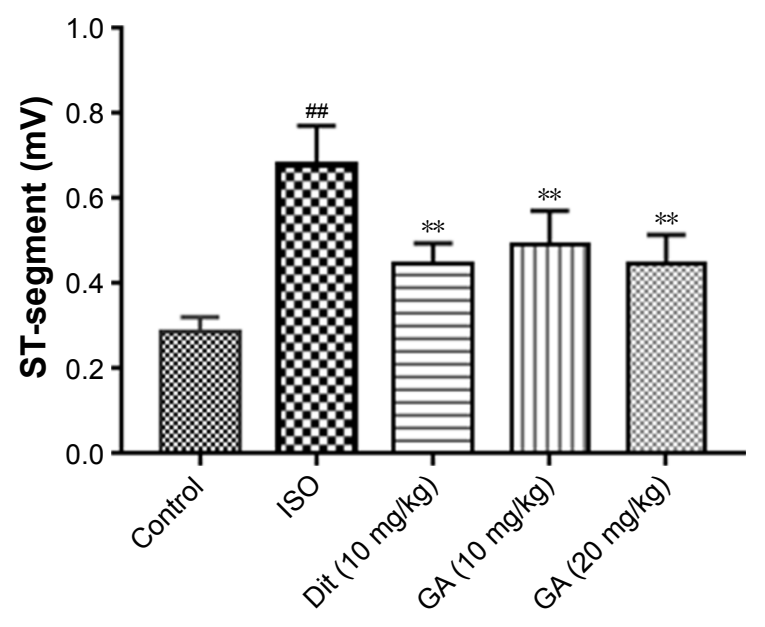

Figure 2 Statistical analysis of GA on ST-segment elevation.

Notes: Values are expressed as mean \pm SD. ${ }^{\#} p<0.0$ I vs control group. ${ }^{* *} p<0.0$ I vs ISO group.

Abbreviations: Dit, diltiazem; GA, glycyrrhizic acid; ISO, isoproterenol; SD, standard deviation.

structure, normal cardiac muscle fibers, and no necrosis. However, compared with the control group, the model group demonstrated abnormal morphological changes which the occurrence of suggested myocardial injury. These changes included myocardial cell swelling, degeneration, transverse striation loss, and inflammatory cells infiltration. The myocardial damage in the GA groups was less than that in the model group. Cardiac sections obtained from rats undergoing GA treatment presented a relatively more intact and less myocardial fiber disruption, necrosis, and inflammatory cell infiltration compared with those from the model group. The histopathological results confirmed that GA could attenuate ISO-induced $\mathrm{MI}$ in rats.

\section{Effects of GA on the expression of proteins in Nrf-2/HO-I and NF-KB pathways}

Nrf2, HO-1, and NF- $\mathrm{KB}$ signaling are the well-known cytoprotective target mediating oxidative stress and inflammation. To clarify whether the Nrf-2/HO-1 and NF- $\kappa$ B pathways were involved in the cardioprotective effect of GA in MI, the expression level of proteins related to Nrf2, HO-1, and $\mathrm{NF}-\kappa \mathrm{B}$ signaling in the ischemic areas of myocardial tissue were detected by Western blots. As revealed in Figure 6, exposure to ISO notably inhibited the protein expressions
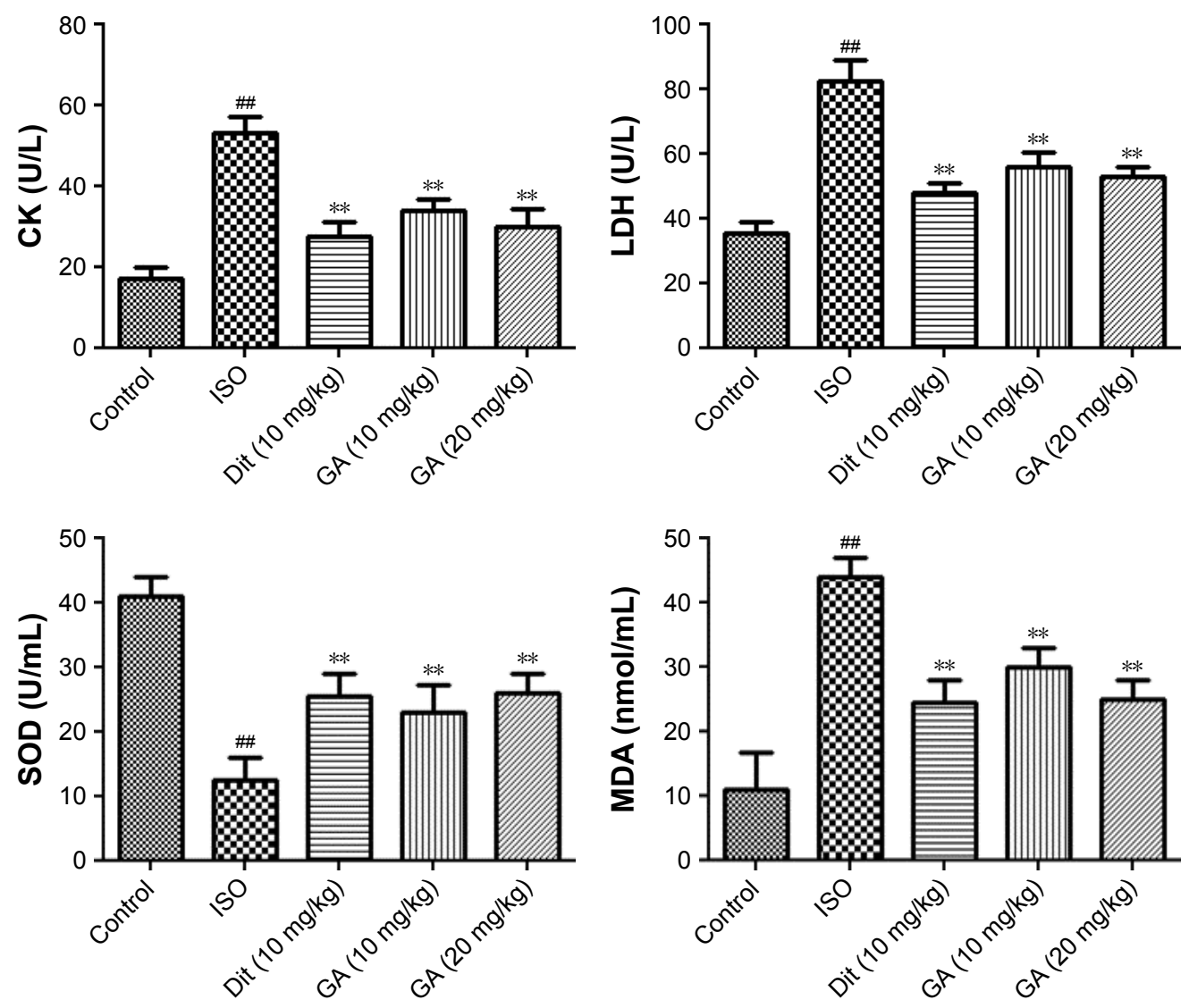

Figure 3 Effects of GA on serum levels of CK, LDH, SOD, and MDA.

Notes: Values represent the mean \pm SD and are representative of two independent experiments. ${ }^{\#} p<0.0$ I, vs control group; ${ }^{* *} p<0.0$ I vs ISO group.

Abbreviations: CK, creatin kinase; Dit, diltiazem; GA, glycyrrhizic acid; LDH, lactate dehydrogenase; ISO, isoproterenol; MDA, malondialdehyde; SD, standard deviation; SOD, superoxide dismutase. 

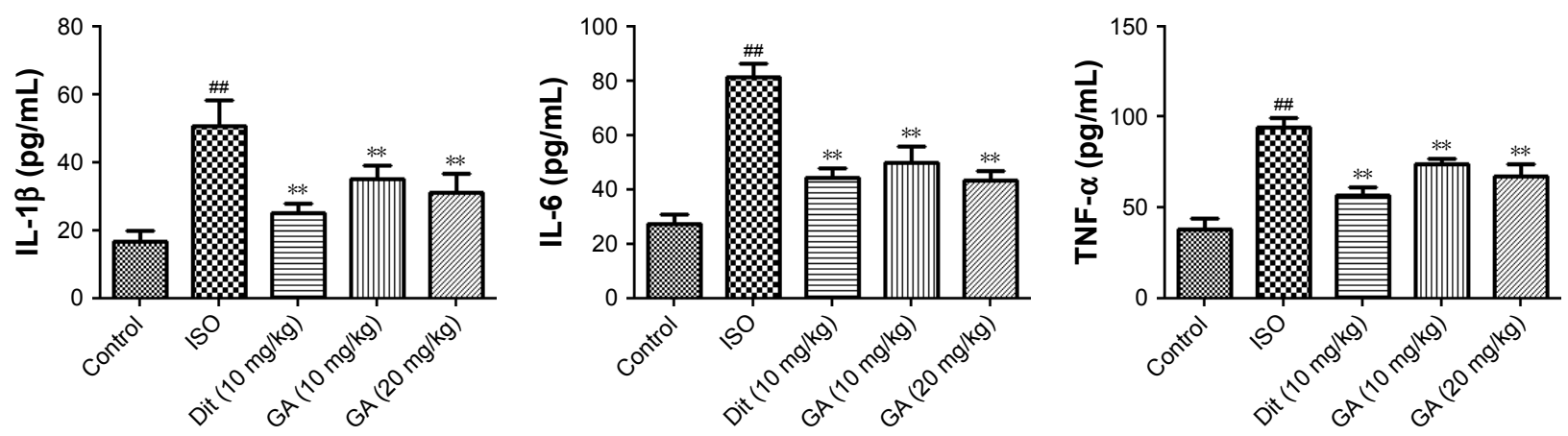

Figure 4 Effects of GA on serum proinflammatory cytokines.

Notes: ${ }^{\#} p<0.0$ I, vs control group; ${ }^{* *} p<0.0$ I vs ISO group.

Abbreviations: Dit, diltiazem; GA, glycyrrhizic acid; ISO, isoproterenol; SD, standard deviation.

of HO-1 and Nrf2. However, after treating with GA (10 and $20 \mathrm{mg} / \mathrm{kg}$ ) and Dit $(10 \mathrm{mg} / \mathrm{kg})$, the protein expressions of HO-1 and Nrf2 was significantly enhanced compared with those in ISO group. In addition, ISO challenge contributed to the upregulations of phosphorylated $\mathrm{I} \kappa \mathrm{B}$ and $\mathrm{NF}-\kappa \mathrm{B}$, while the treatment with GA (10 and $20 \mathrm{mg} / \mathrm{kg}$ ) and Dit $(10 \mathrm{mg} / \mathrm{kg}$ ) effectively downregulated the levels of $\mathrm{p}$-IкB and $\mathrm{p}-\mathrm{NF}-\kappa \mathrm{B}$.

\section{Discussion}

MI remains the large cause of morbidity and mortality for an increasing proportion of the world's population.
Evidence has emerged indicating that MI injury result due to hypoxia is linked with diminished energy supply and oxidative stress. ${ }^{15}$ ISO is a synthetic $\beta$-adrenergic agonist that can cause increased myocardial oxygen consumption and necrosis of heart muscle cells. ${ }^{16}$ Large doses of ISO administered in rats can induce acute MI. So in the current study, we build the acute MI induced by ISO. The acute MI induced by ISO was confirmed by ST-segment elevation, loss of integrity of myocardial membranes on histological examination, and serum elevation of CK and LDH enzymes. Pretreatment with GA reduced the ST-segment elevation induced by acute MI and alleviated myocardial ischemic

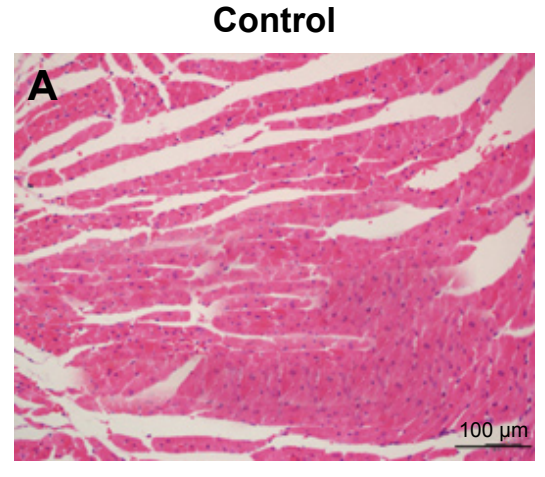

\section{GA (10 mg/kg)}

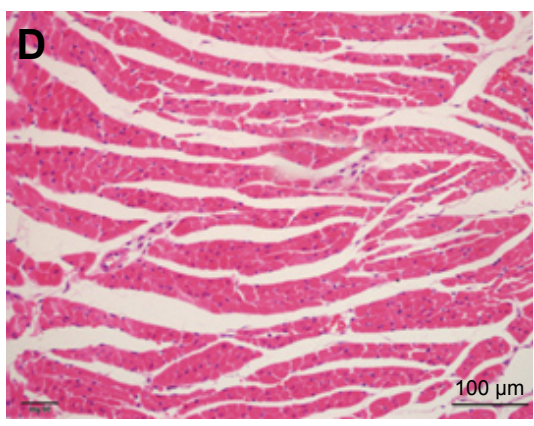

ISO

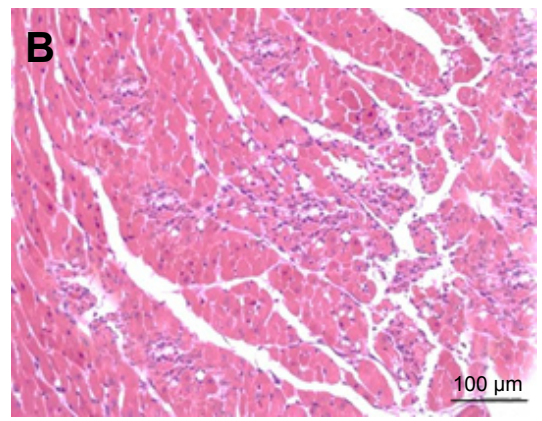

Dit $(10 \mathrm{mg} / \mathrm{kg})$

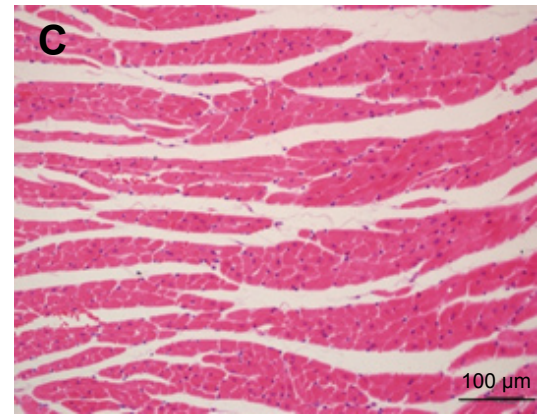

GA $(20 \mathrm{mg} / \mathrm{kg})$

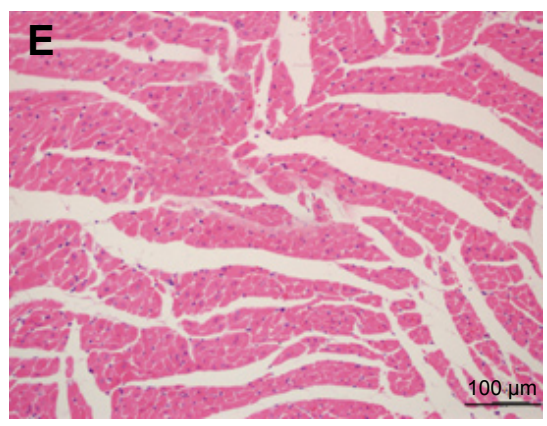

Figure 5 Effects of GA on histopathological lesions.

Notes: (A) control group, (B) model (ISO) group, (C) the ISO+Dit (10 mg/kg) group, (D) ISO+GA (I0 mg/kg) group, and (E) ISO+GA (20 mg/kg) group (hematoxylin and eosin staining, magnification 200x).

Abbreviations: Dit, diltiazem; GA, glycyrrhizic acid; ISO, isoproterenol. 

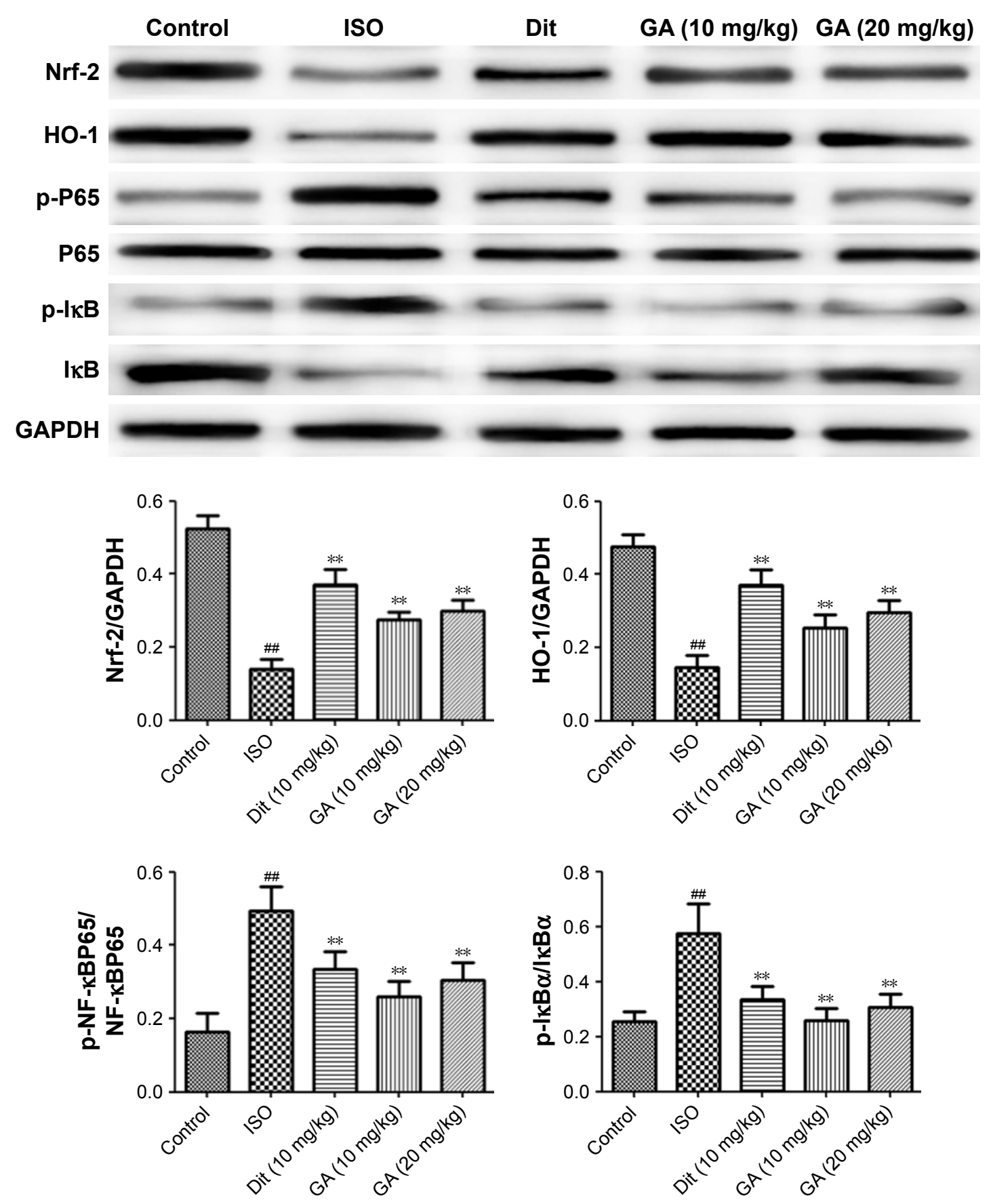

Figure 6 Effects of GA on Nrf-2/HO-I pathway-related proteins in rats.

Notes: Values are expressed as mean \pm SD. ${ }^{*} p<0.0$ I vs control group. ${ }^{* *} p<0.0$ I vs ISO group.

Abbreviations: Dit, diltiazem; GA, glycyrrhizic acid; ISO, isoproterenol; SD, standard deviation.

injury. GA also decreased CK, LDH, MDA, IL-1 $\beta$, TNF- $\alpha$, and IL-6 levels, and increased SOD activity. These results suggested that GA in the doses used in this study exhibited cardioprotective effects in MI, which could be attributed to its antioxidative and anti-inflammatory properties.

Free radical generation and consequent oxidative stress in ischemia leads to tissue damage, which is accompanied by activation of the inflammatory responses. Previous research has demonstrated that oxidative stress is one of the key mechanisms playing a role in the process of myocardial ischemic injury. ${ }^{17}$ Oxidative stress can damage cellular macromolecules, inducing protein modification, lipid peroxidation, as well as DNA damage. ${ }^{18}$ The levels of SOD and MDA activity are two principal pathophysiological parameters for evaluating free radical metabolism. SOD is an important antioxidant enzyme to alleviate myocardial injury and reflects the cellular capability of scavenging/quenching free radicals. ${ }^{19}$ In this study, SOD activity was significantly 
decreased in the ISO-treated rats as compared to controls. Administration of GA significantly restored SOD activity and reduced the content of MDA, which suggested that the cardioprotective effect of GA were related to antioxidative properties.

Previous studies showed that excessive oxidative stress contributed to overexpressions of multiple cytokines, such as TNF- $\alpha$, IL-6, and IL-1 $\beta$. The elevated levels of inflammatory markers were associated with MI. ${ }^{20}$ IL-6, released from vascular endothelial cells, aggravates the progress of inflammatory response and inhibits infiltration of neutrophils. ${ }^{21}$ During inflammatory disease procedures, TNF- $\alpha$ and IL- $1 \beta$ are pleiotropic to control rheumatoid arthritis disease progression. The elevation of IL-1 $\beta$ promoted the apoptosis of cardiomyocytes and upregulated the expression of matrix metalloproteinases. $^{22}$ Thus, TNF- $\alpha$, IL-6, and IL-1 $\beta$ are important inflammatory mediators in regulating inflammatory response procedures. In this study, the inflammatory stresses induced by ISO were reflected by serum IL-6, IL-1 $\beta$, and TNF- $\alpha$ elevations. GA preconditioning decreased the levels of proinflammatory cytokines, which suggested that the cardioprotective effects of GA were also associated with anti-inflammatory properties.

In MI injury, oxidative stress is induced by an excess of intracellular oxidants or ROS. Since endogenous antioxidant proteins can eliminate intracellular oxidants and ROS, the antioxidant proteins play an important role in antioxidant stress. ${ }^{23}$ Therefore, improving the expression of endogenous antioxidant proteins could be an effective strategy to reduce myocardial cell damage. Recent evidence indicates that Nrf2 is a key regulator of endogenous antioxidant systems. ${ }^{24} \mathrm{Nrf}-2$ activates endogenous antioxidant enzymes to reduce oxidative stress. During the procedures, Nrf-2 dissociates from Keap1, binds to the ARE, and activates phase II enzymes such as HO- $1 . .^{25,26}$ The rate-limiting enzyme HO-1 is considered as a stress protein, which is a sensitive indicator of oxidative stress in the myocardium. MI also results in significant cardiac dysfunction owing to the increase of inflammation-related genes expressions. NF- $\kappa \mathrm{B}$ has been identified to be a pivotal regulator of inflammatory signaling pathways to produce proinflammatory cytokines. ${ }^{27}$ In an inactive state, NF- $\kappa \mathrm{B}$ resides in the cytoplasm, where it associates with an inhibitory

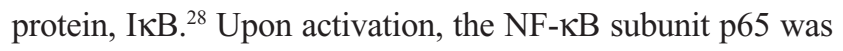
promoted via the phosphorylation and degradation of the I $\mathrm{I} B{ }^{29}$ In the current study, we discussed the interlinkage between Nrf-2 and NF- $\kappa$ B. The activation of Nrf-2 was accompanied by the production of HO-1. As a key mediator in inflammation response, NF- $\kappa \mathrm{B}$ promoted the elevation of nitric oxide after its activation. Abass et $\mathrm{al}^{31}$ previously found that Nrf-2 upregulation was related to the inhibition of NF- $\kappa \mathrm{B}$ translocation by $\mathrm{HO}-1$ end products, including bilirubin and $\mathrm{CO} .{ }^{30}$ Although GA has been reported previously to relieve cardiac ischemia in rats, ${ }^{13}$ it was not clear that whether GA attenuated MI injury through regulating Nrf-2 and NF- $\mathrm{BB}$ pathways. To further explore the mechanisms of GA protective effect on MI rats, we examined the effects of GA on the activation of the Nrf-2/ $\mathrm{HO}-1$ and NF- $\kappa \mathrm{B}$ signaling pathways. The Western blot data displayed that GA treatment effectively upregulated HO-1 and $\mathrm{Nrf} 2$ expressions and downregulated the phosphorylation of $\mathrm{I} \kappa \mathrm{B}$ and $\mathrm{NF}-\kappa \mathrm{B}$ caused by ISO challenge. In Cao and Wu's work, GA showed activities as inducers of Nrf2 and inhibitors of NF- $\kappa \mathrm{B}$ activation in the liver and the kidney. To the best of our knowledge, our work is the first to study the regulation of GA on Nrf-2 and NF- $\kappa$ B pathways in myocardial tissue. Taken together, the current data confirmed that GA exhibited remarkable protective effects on ISO-induced MI through suppressing oxidative stress and inflammatory cytokines. In addition, GA could activate the Nrf2 antioxidant response and inhibit NF- $\kappa$ B activation, which may be partly associated with its protective effect in ISO-induced oxidative stress and inflammatory responses. These findings suggest that GA could be developed as potential, efficient therapeutic drug against MI in the future. However, further, more comprehensive studies are warranted to explore its future clinical application.

\section{Acknowledgment}

The study was supported by the Priority Academic Program Development of Jiangsu Higher Education Institutions (PAPD).

\section{Author contributions}

All authors contributed toward data analysis, drafting and revising the paper and agree to be accountable for all aspects of the work.

\section{Disclosure}

The authors report no conflicts of interest in this work.

\section{References}

1. Thom T, Haase N, Rosamond W, et al. Heart disease and stroke statistics2006 update: a report from the American Heart Association Statistics Committee and Stroke Statistics Subcommittee. Circulation. 2006;113: e85-e151.

2. Mackay J, Mensah GA, Mendis S, Greenlund K. The Atlas of Heart Disease and Stroke. 1st ed. Geneva: World Health Organization; 2004.

3. Benjamin EJ, Blaha MJ, Chiuve SE, et al. Heart disease and stroke statistics - 2017 update: a report from the American Heart Association. Circulation. 2017;135:e146-e603. 
4. Songco AV, Brener SJ. Initial strategy of revascularization versus optimal medical therapy for improving outcomes in ischemic heart disease: a review of the literature. Curr Cardiol Rep. 2012;14:397-407.

5. Brevoord D, Kranke P, Kuijpers M, Weber N, Hollmann M, Preckel B. Remote ischemic conditioning to protect against ischemia-reperfusion injury: a systematic review and meta-analysis. PLoS One. 2012;7: e42179.

6. Tzanavari T, Varela A, Theocharis S, et al. Metformin protects against infection-induced myocardial dysfunction. Metabolism. 2016;65: 1447-1458.

7. Ferrari RS, Andrade CF. Oxidative stress lung ischemia-reperfusion injury. Oxid Med Cell Longev. 2015;2015:590987.

8. Aravinthan A, Kim JH, Antonisamy P, et al. Ginseng total saponin attenuates myocardial injury via anti-oxidative and anti-inflammatory properties. J Ginseng Res. 2015;39:206-212.

9. Yu J, Zhang W, Zhang R, et al. Molecular hydrogen attenuates hypoxia/ reoxygenation injury of intrahepatic cholangiocytes by activating $\mathrm{Nrf} 2$ expression. Toxicol Lett. 2015;238:11-19.

10. Yu Q, Li X, Cao X. Linarin could protect myocardial tissue from the injury of ischemia-reperfusion through activating Nrf-2. Biomed Pharmacother. 2017;90:1-7.

11. Su X, Wu L, Hu M, Dong W, Xu M, Zhang P. Glycyrrhizic acid: a promising carrier material for anticancer therapy. Biomed Pharmacother. 2017;95:670-678.

12. Kim YM, Kim HJ, Chang KC. Glycyrrhizin reduces HMGB1 secretion in lipopolysaccharide-activated RAW 264.7 cells and endotoxemic mice by $\mathrm{p} 38 / \mathrm{Nrf2}$-dependent induction of HO-1. Int Immunopharmacol. 2015;26:112-118.

13. Haleagrahara N, Varkkey J, Chakravarthi S. Cardioprotective effects of glycyrrhizic acid against isoproterenol-induced myocardial ischemia in rats. Int J Mol Sci. 2011;12:7100-7113.

14. Xu MF, Wei SS, Qin XW, Zhang YT, Liu CY. Rule-based method for morphological classification of ST segment in ECG signals. J Med Biol Eng. 2015;35:816-823.

15. Chang XY, Zhang K, Zhou R, et al. Cardioprotective effects of salidroside on myocardial ischemia-reperfusion injury in coronary artery occlusion-induced rats and Langendorff-perfused rat hearts. Int J Cardiol. 2016;215:532-544.

16. Moris D, Spartalis M, Tzatzaki E, et al. The role of reactive oxygen species in myocardial redox signaling and regulation. Ann Transl Med. 2017;5:324.

17. Zhu L, Wei T, Chang X, et al. Effects of salidroside on myocardial injury in vivo in vitro via regulation of Nox/NF- $\mathrm{BB} / \mathrm{AP} 1$ pathway. Inflammation. 2015;38:1589-1598.
18. Kurian GA, Rajagopal R, Vedantham S, Rajesh M. The role of oxidative stress in myocardial ischemia and reperfusion injury and remodeling: revisited. Oxid Med Cell Longev. 2016;2016:1656450.

19. Rathore KI, Kerr BJ, Redensek A, et al. Ceruloplasmin protects injured spinal cord from iron-mediated oxidative damage. J Neurosci. 2008; 28:12736-12747.

20. Jiang W, Zhou R, Li P, et al. Protective effect of chrysophanol on LPS/d-

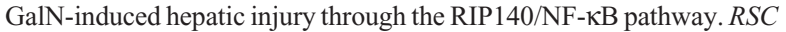
Adv. 2016;6:38192-38200.

21. He H, Chang X, Gao J, Zhu L, Miao M, Yan T. Salidroside mitigates sepsis-induced myocarditis in rats by regulating IGF-1/PI3K/Akt/ GSK-3 $\beta$ signaling. Inflammation. 2015;38:2178-2184.

22. Mirzaei H, Ferns GA, Avan A, Mobarhan MG. Cytokines and microRNA in coronary artery disease. Adv Clin Chem. 2017;82:47-70.

23. Akolkar G, da Silva Dias D, Ayyappan P, et al. Vitamin C mitigates oxidative/nitrosative stress and inflammation in doxorubicin-induced cardiomyopathy. Am J Physiol Heart Circ Physiol. 2017;313: H795-H809.

24. Lim JH, Lee YM, Chun YS, Chen J, Kim JE, Park JW. Sirtuin 1 modulates cellular responses to hypoxia by deacetylating hypoxia-inducible factor 1 $\alpha$. Mol Cell. 2010;38:864-878.

25. Chang X, Zhang K, Zhou R, et al. Cardioprotective effects of salidroside on myocardial ischemia-reperfusion injury in coronary artery occlusion-induced rats and Langendorff-perfused rat hearts. Int $J$ Cardiol. 2016;215:532-544.

26. Shah ZA, Li RC, Thimmulappa RK, et al. Role of reactive oxygen species in modulation of Nrf2 following ischemic reperfusion injury. Neuroscience. 2007;147:53-59.

27. Kaspar JW, Niture SK, Jaiswal AK. Nrf2:INrf2 (Keap1) signaling in oxidative stress. Free Radic Biol Med. 2009;47:1304-1309.

28. Chen T, Mou Y, Tan J, et al. The protective effect of CDDO-Me on lipopolysaccharide-induced acute lung injury in mice. Int Immunopharmacol. 2015;25:55-64.

29. Jiang W, Zhou R, Li P, et al. Protective effect of chrysophanol on LPS/d-

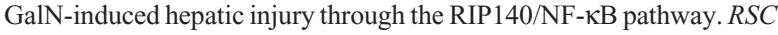
Adv. 2016;6:38192-38200.

30. Zhang X, Du Q, Yang Y, Wang J, Dou S, Liu C. The protective effect of Lutelin on myocardial ischemia/reperfusion (IR) injury through TLR4/NF-Kb/NLRP3 inflammasome pathway. Biomed Pharmacother. 2017;91:1042-1052.

31. Abass MA, Elkhateeb SA, Abd El-Baset SA, Kattaia AA, MohamedEM, Atteia $\mathrm{HH}$. Lycopene ameliorates atrazine-induced oxidative damage in adrenal cortex of male rats by activation of the Nrf2/HO-1 pathway. Environ Sci Pollut Res. 2016;23:15262.

\section{Publish your work in this journal}

Drug Design, Development and Therapy is an international, peerreviewed open-access journal that spans the spectrum of drug design and development through to clinical applications. Clinical outcomes, patient safety, and programs for the development and effective, safe, and sustained use of medicines are the features of the journal, which

\section{Dovepress}

has also been accepted for indexing on PubMed Central. The manuscript management system is completely online and includes a very quick and fair peer-review system, which is all easy to use. Visit http://www.dovepress.com/testimonials.php to read real quotes from published authors. 